\title{
Lie Symmetry Reductions and Exact Solutions of a Multidimensional Double Dispersion Equation
}

\author{
Jiali Yu' ${ }^{1}$, Fuzhi Li' ${ }^{2}$, Lianbing She ${ }^{3}$ \\ ${ }^{1}$ School of Mathematics and Information Science, Guangzhou University, Guangzhou, China \\ ${ }^{2}$ School of Mathematics and Statistics, Southwest University, Chongqing, China \\ ${ }^{3}$ Department of Mathematics, Southwest University, Chongqing, China \\ Email: jialiyu97@yahoo.com, lifuzhifight@foxmail.com, shelianbing@163.com
}

How to cite this paper: Yu, J.L., Li, F.Z. and She, L.B. (2017) Lie Symmetry Reductions and Exact Solutions of a Multidimensional Double Dispersion Equation. Applied Mathematics, 8, 712-723. https://doi.org/10.4236/am.2017.85056

Received: April 16, 2017

Accepted: May 22, 2017

Published: May 25, 2017

Copyright $\odot 2017$ by authors and Scientific Research Publishing Inc. This work is licensed under the Creative Commons Attribution International License (CC BY 4.0).

http://creativecommons.org/licenses/by/4.0/ c) (i) Open Access

\begin{abstract}
In this paper, based on classical Lie group method, we study a multidimensional double dispersion equation, and get its infinitesimal generator, symmetry group and similarity reductions. In particular, similarity solutions and travelling wave solutions of the multidimensional double dispersion equation are derived from the reduction equations.
\end{abstract}

\section{Keywords}

Lie Group, Multidimensional Double Dispersion Equation, Similarity Solutions, Travelling Wave Solutions

\section{Introduction}

The double dispersion Equation (1) was introduced as a mathematical model of nonlinear dispersive waves in various contexts (see [1] [2] [3] [4])

$$
u_{t t}-u_{x x}+a u_{x x x x}-b u_{x x t t}=f(u)_{x x},
$$

where $u=u(x, t)$ is a real-valued function, $a, b$ are positive real constants with $a \geq b$. It also presents the plots of the instability/stability regions of travelling waves for various values of $p$, where $f(u)=|u|^{p-1} u, p>1$. The present paper provides an overview of results obtained in [5] and [6] for travelling wave solutions of the Equation (1).

Considering the possibility of energy exchange through lateral surfaces of the waveguide in the physical study of nonlinear wave propagation in waveguide, the longitudinal displacement $u(x, t)$ of the rod satisfies the following double dispersion equation (DDE) (see [7] [8] [9]) 


$$
u_{t t}-u_{x x}=\frac{1}{4}\left(6 u^{2}+a u_{t t}-b u_{x x}\right)_{x x},
$$

and the general cubic DDE (CDDE)

$$
u_{t t}-u_{x x}=\frac{1}{4}\left(c u^{3}+6 u^{2}+a u_{t t}-b u_{x x}+d u_{t}\right)_{x x},
$$

where $a, b$ and $c$ are positive constants. The Equations (2) and (3) were studied in some literatures, the travelling wave solutions, depending upon the phase variable $Z=X \pm c t$ were studied by Samsonov in [10] [11].

In [12] [13], Chen and Wang studied the initial-boundary value problem and the Cauchy problem of the following generalized double dispersion equation which includes above Equation (3) as special cases

$$
u_{t t}-u_{x x}-a u_{x x t t}+b u_{x x x x}-d u_{x x t}=f(u)_{x x},
$$

where $a>0, b>0$ and $d$ are constants.

Recently in [14], the authors considered the Cauchy problem of the multidimensional nonlinear evolution equation

$$
\begin{aligned}
& u_{t t}-\Delta u-\Delta u_{t t}+\Delta^{2} u-k \Delta u_{t}=\Delta f(u), x \in R^{n}, t>0, \\
& u(x, 0)=u_{0}(x), u_{t}(x, 0)=u_{1}(x), x \in R^{n}
\end{aligned}
$$

where $k$ is an arbitrary real constant. The authors gave the existence of local solution and the existence of global solution. And in [15], Zhijian Yang et al. studied the existence of global attractor for the generalized double dispersion equation arising in elastic waveguide model. And there have been lots of research studies on the well-posedness, blowup, asymptotic behavior and other properties of solutions for both the IBVP and the IVP of the equation of type (6) (see [16]-[24]) and references therein). While for the investigation on the global attractor to Equation (6), one can see [25] [26] [27] and references therein.

Symmetry reductions have several important applications in the context of differential equations. Since solutions of partial differential equations asymptotically tend to solutions of equations obtained by symmetry reduction, some of these special solutions will illustrate important physical phenomena (see [28] [29] [30] [31] [32]). Solitary wave solutions and similarity solutions are usually applied to describe physical phenomena and to check on the accuracy and reliability of numerical algorithm, so deriving travelling wave solutions and similarity solutions has a great significance (see [33] [34] [35]).

In [36], the authors applied the method of Lie and the nonclassical method of Bluman and Cole to undertake the following equation

$$
u_{x x x x}+f(u)_{x x}=u_{t t},
$$

In [37], the authors applied the Lie-group formalism to deduce symmetries of a generalized double dispersion equation

$$
u_{t t}-u_{x x}+u_{x x x x}-u_{x x t t}-\left(u^{n}\right)_{x x}=0
$$

and they obtained exact solutions which can be expressed by various single and 
combined nondegenerative Jacobi elliptic function solutions.

In [38], the authors consider the generalized double dispersion equation

$$
u_{t t}-u_{x x}-a u_{x x t t}+b u_{x x x x}-d u_{x x t}-f(u)_{x x}=0 \text {, }
$$

where $a>0, b>0$ and $d$ are constants. They study the functional forms $f(u)$ for which Equation (9) with $a, b \neq 0$ admits classical symmetries.

In this paper, we consider the following multidimensional double dispersion equation

$$
u_{t t}-\Delta u-\Delta u_{t t}+\Delta^{2} u+k \Delta u_{t}=\Delta f(u), x \in R^{n}, t>0,
$$

where $n=3, f(u)=|u|^{p}, p>1$ or $f(u)=u^{2 k}, k=1,2, \cdots$,

In this paper, the symmetry group of the $n$-dimensional double dispersion Equation (10) is obtained by using the classical method in Section 2. In Section 3, we discuss the Lie symmetry group of Equation (10). Finally, we obtain similarity solutions or travelling wave solutions of Equation (10) by using similarity variables to obtain reduction equations, and solving the reduction equations in Section 4.

\section{Lie Symmetry Analysis of the Double Dispersion Equation}

In this section, we perform Lie symmetry analysis for Equation (10), and obtain its infinitesimal generator.

Theorem 1. [28] Assume

$$
\underline{V}=\sum_{i=1}^{p} \xi^{i}(x, u) \frac{\partial}{\partial x^{i}}+\sum_{\alpha=1}^{p} \phi_{\alpha}(x, u) \frac{\partial}{\partial x^{\alpha}},
$$

be a vector field on $X \times U$, where $X=\left(x^{1}, x^{2}, \cdots, x^{p}\right), U=\left(u^{1}, u^{2}, \cdots, u^{q}\right)$. Then its n-st prolongation is defined a vector on $X \times U$

$$
p r^{(n)} \underline{V}=\underline{V}+\sum_{\alpha=1}^{p} \sum_{J=1} \phi_{\alpha}^{J}\left(x, u^{(n)}\right) \frac{\partial}{\partial u_{J}^{\alpha}},
$$

where, by definition

$$
\phi_{\alpha}^{J}\left(x, u^{(n)}\right)=D_{J} Q_{\alpha}+\sum_{i=1}^{p} \xi^{i} u_{J i}^{\alpha}, J=\left(j_{1}, \cdots, j_{l}\right), 0 \leq k \leq n, 1 \leq j_{k} \leq p,
$$

where, $Q_{\alpha}=\phi_{\alpha}(x, u)-\sum_{i=1}^{p} \xi^{i}(x, u) \frac{\partial}{\partial x^{i} u_{i}^{\alpha}}, \quad \alpha=1, \cdots, q, Q(x, u)=\left(Q_{1}, \cdots, Q_{q}\right)$ is referred to as the characteristic of the vector field $\underline{V}$.

Here are four independent variables $x, y, z$ being spatial coordinates and the time. According to the method of determining the infinitesimal generator of nonlinear partial differential equation [28], we take the infinitesimal generator of equation as follows:

$$
\begin{aligned}
\underline{V}= & \xi(x, y, z, t, u) \frac{\partial}{\partial x}+\eta(x, y, z, t, u) \frac{\partial}{\partial y}+\zeta(x, y, z, t, u) \frac{\partial}{\partial z} \\
& +\tau(x, y, z, t, u) \frac{\partial}{\partial t}+\phi(x, y, z, t, u) \frac{\partial}{\partial u},
\end{aligned}
$$

be a vector field on $X \times U$. Where $\xi, \eta, \zeta, \tau, \phi$ are coefficient functions of the infinitesimal generator to be determined. We wish to determine all possible coe- 
fficient functions $\xi, \eta, \zeta, \tau$ and $\phi$ so that the corresponding one-parameter group $\exp (\varepsilon \underline{V})$ is a symmetry group of the double dispersion equation. Applying the forth prolongation of $\underline{V}$ to Equation (10), we find the invariance condition $\left.\operatorname{pr}^{(4)} \underline{V}(\Delta)\right|_{\Delta}=0$, where $\Delta$ is $u_{t t}-\Delta u-\Delta u_{t t}+\Delta^{2} u+k \Delta u_{t}-\Delta\left(u^{p}\right)$ and with help of Maple software, we find the following system of symmetry equations

$$
\begin{aligned}
& \phi^{t t}-\left(\phi^{x x}+\phi^{y y}+\phi^{z z}\right)-\left(\phi^{x x t t}+\phi^{y y t t}+\phi^{z z t t}\right) \\
& +\left(\phi^{x x x x}+\phi^{y y y y}+\phi^{z z z z}\right)+k\left(\phi^{x x t}+\phi^{y y t}+\phi^{z z t}\right) \\
& =p(p-1)(p-2) u^{p-3}\left(u_{x}^{2}+u_{y}^{2}+u_{z}^{2}\right) \phi \\
& +p(p-1) u^{p-2}\left(2 \nabla u \phi^{x}+\Delta u \phi\right)+p u^{p-1}\left(\phi^{x x}+\phi^{y y}+\phi^{z z}\right),
\end{aligned}
$$

which must be satisfied whenever $u$ satisfy Equation (10). Here $\phi^{t t}, \phi^{x x}$, etc. are the coefficients of the second order derivatives $\frac{\partial}{\partial u_{t t}}, \frac{\partial}{\partial u_{x x}}$, etc. appearing in $p r^{(4)} \underline{V}$.

According to Th. $1, \phi_{\alpha}^{J}\left(x, u^{(n)}\right)=D_{J}\left(\phi_{\alpha}-\Sigma \xi^{i} u_{i}^{\alpha}\right)+\Sigma \xi^{i} u_{J, i}^{\alpha}$, we have

$$
\begin{aligned}
\phi^{x x}= & D_{x}^{2} \phi-u_{x} D_{x}^{2} \xi-u_{y} D_{x}^{2} \eta-u_{z} D_{x}^{2} \varsigma-u_{t} D_{x}^{2} \tau \\
& -2 u_{x x} D_{x} \xi-2 u_{x y} D_{x} \eta-2 u_{x z} D_{x} \varsigma-2 u_{x t} D_{x} \tau .
\end{aligned}
$$

Similarly, we can get $\phi^{t t}, \phi^{x x t}, \phi^{x x x x}$, etc. we find the determining equations for the symmetry group of Equation (10) to be the following:

$$
\left\{\begin{array}{l}
\xi_{u}=\eta_{u}=\zeta_{u}=0, \xi_{t}=\eta_{t}=\zeta_{t}=0, \\
\phi_{u u}=0, \\
\tau_{x}=\tau_{y}=\tau_{z}=\tau_{u}=0, \\
\left(\xi_{x}+\eta_{y}+\zeta_{z}\right)-\tau_{t}=0, \\
2 \nabla \phi_{u}-3\left(\xi_{x x}+\eta_{y y}+\zeta_{z z}\right)=0, \\
2 \nabla \phi_{u}-\left(\xi_{x x}+\eta_{y y}+\zeta_{z z}\right)=0, \\
\Delta \phi_{u}-2\left(\xi_{x}+\eta_{y}+\zeta_{z}\right)=0, \\
2 \phi_{t u}-k \tau_{t}-\tau_{t t}=0, \\
\nabla \phi_{t u}+k\left(\xi_{x x}+\eta_{y y}+\zeta_{z z}\right)-2 k \nabla \phi_{u}=0, \\
k \Delta \phi_{u}-2 \Delta \phi_{t}+2 \phi_{t u}-\tau_{t t}=0, \\
u^{p-3}\left[u \phi_{u}+(p-2) \phi+2 \tau_{t} u\right]=0, \\
-6 \Delta \phi_{u}+\phi_{t t u}-k \phi_{t u}+p(p-1) u^{p-2} \eta+2 p u^{p-1} \tau_{t}=0, \\
\Delta^{2} \phi-p u^{p-1} \Delta \phi-\Delta \phi-\Delta \phi_{t t}+\phi_{t t}+k \Delta \phi_{t}=0, \\
2 p(p-1) u^{p-2} \nabla \phi-4 \nabla \Delta \phi_{u}+2 p u^{p-1} \nabla \phi_{u}+2 \nabla \phi_{u}+2 \nabla \phi_{t t u}-2 k \nabla \phi_{t u} \\
-p u^{p-1}\left(\xi_{x x}+\eta_{y y}+\zeta_{z z}\right)+\left(\xi_{x x x x}+\eta_{y y y y}+\zeta_{z z z z}\right)-\left(\xi_{x x}+\eta_{y y}+\zeta_{z z}\right)=0,
\end{array}\right.
$$

Since we have now satisfied all the determining equations, we conclude that general infinitesimal symmetry of Equation (10) has coefficient functions of the following form:

$$
\xi=c_{1},
$$




$$
\begin{aligned}
& \eta=c_{2}, \\
& \zeta=c_{3}, \\
& \tau=c_{4},
\end{aligned}
$$

where $c_{1}, \cdots, c_{4}$ are arbitrary constants. Thus the Lie-algebra of infinitesimal of the double dispersion equation is spanned by four vector fields:

$$
\begin{aligned}
& V_{1}=\frac{\partial}{\partial x}, \\
& V_{2}=\frac{\partial}{\partial y}, \\
& V_{3}=\frac{\partial}{\partial z}, \\
& V_{4}=\frac{\partial}{\partial t},
\end{aligned}
$$

so we have

$$
\underline{V}=c_{1} V_{1}+c_{2} V_{2}+c_{3} V_{3}+c_{4} V_{4}
$$

\section{Symmetry Groups of the Double Dispersion Equation}

In this section, in order to get some exact solutions from a known solution of Equation (10), we should find the one-parameter symmetry groups $g_{i}:(x, y, z, y, u) \rightarrow(\bar{x}, \bar{y}, \bar{z}, \bar{t}, \bar{u})$ of corresponding infinitesimal generators. To get the Lie symmetry groups, we should solve the following initial problems of ordinary differential equations:

$$
\left\{\begin{array}{l}
\frac{\mathrm{d}(\bar{x}, \bar{y}, \bar{z}, \bar{t}, \bar{u})}{\mathrm{d} \varepsilon}=(\xi, \eta, \zeta, \tau, \phi) \\
\left.(\bar{x}, \bar{y}, \bar{z}, \bar{t}, \bar{u})\right|_{\varepsilon=0}=(x, y, z, t, u),
\end{array}\right.
$$

where

$$
\begin{aligned}
\xi & =\xi(\bar{x}, \bar{y}, \bar{z}, \bar{t}, \bar{u}), \\
\eta & =\eta(\bar{x}, \bar{y}, \bar{z}, \bar{t}, \bar{u}), \\
\zeta & =\zeta(\bar{x}, \bar{y}, \bar{z}, \bar{t}, \bar{u}), \\
\tau & =\tau(\bar{x}, \bar{y}, \bar{z}, \bar{t}, \bar{u}), \\
\phi & =\phi(\bar{x}, \bar{y}, \bar{z}, \bar{t}, \bar{u})
\end{aligned}
$$

and $\varepsilon$ is a group parameter.

For the infinitesimal generator $\underline{V}=c_{1} V_{1}+c_{2} V_{2}+c_{3} V_{3}+c_{4} V_{4}$, we will take the following different values to obtain the corresponding infinitesimal generators:

Case 1. $C_{1}=1, C_{2}=C_{3}=C_{4}=0$, the infinitesimal generator is $V_{1}=\frac{\partial}{\partial x}$,

Case 2. $C_{2}=1, C_{1}=C_{3}=C_{4}=0$, the infinitesimal generator is $V_{2}=\frac{\partial}{\partial y}$,

Case 3. $C_{3}=1, C_{1}=C_{2}=C_{4}=0$, the infinitesimal generator is $V_{3}=\frac{\partial}{\partial z}$, 
Case 4. $C_{4}=1, C_{1}=C_{2}=C_{3}=0$, the infinitesimal generator is $V_{4}=\frac{\partial}{\partial t}$,

Case 5. $C_{1}=C_{2}=C_{3}=1, C_{4}=0$, the infinitesimal generator is $V_{5}=V_{1}+V_{2}+V_{3}=\frac{\partial}{\partial x}+\frac{\partial}{\partial y}+\frac{\partial}{\partial z}$,

Case 6. $C_{1}=C_{2}=C_{3}=C_{4}=1$, the infinitesimal generator is $V_{6}=V_{1}+V_{2}+V_{3}+V_{4}=\frac{\partial}{\partial x}+\frac{\partial}{\partial y}+\frac{\partial}{\partial z}+\frac{\partial}{\partial t}$,

Case 7. $C_{1}=C_{2}=C_{3}=\lambda, C_{4}=\beta$, the infinitesimal generator is $V_{7}=V_{1}+V_{2}+V_{3}+V_{4}=\lambda\left(\frac{\partial}{\partial x}+\frac{\partial}{\partial y}+\frac{\partial}{\partial z}\right)+\beta \frac{\partial}{\partial t}$,

The one-parameter groups $G_{i}$ generated by the $V_{i}$. The entries give the transformed point $\exp \left(\varepsilon V_{i}\right)(x, y, z, t, u)=(\bar{x}, \bar{y}, \bar{z}, \bar{t}, \bar{u})$ :

$$
\begin{aligned}
& G_{1}:(x+\varepsilon, y, z, t, u), \\
& G_{2}:(x, y+\varepsilon, z, t, u), \\
& G_{3}:(x, y, z+\varepsilon, t, u), \\
& G_{4}:(x, y, z, t+\varepsilon, u), \\
& G_{5}:(x+\varepsilon, y+\varepsilon, z+\varepsilon, t, u), \\
& G_{6}:(x+\varepsilon, y+\varepsilon, z+\varepsilon, t+\varepsilon, u), \\
& G_{7}:(x+\lambda \varepsilon, y+\lambda \varepsilon, z+\lambda \varepsilon, t+\beta \varepsilon, u),
\end{aligned}
$$

where $G_{1}, G_{2}, G_{3}$ are space translations, $G_{4}$ is a time translation. $\varepsilon$ is an arbitrary constant.

Theorem 2. If $u=f(x, y, z, t)$ is a known solution of Equation (10), then by using the symmetry groups $G_{i}(i=1,2,3,4)$, so are the functions

$$
\begin{aligned}
& u_{1}=f(x-\varepsilon, y, z, t), \\
& u_{2}=f(x, y-\varepsilon, z, t), \\
& u_{3}=f(x, y, z-\varepsilon, t), \\
& u_{4}=f(x, y, z, t-\varepsilon), \\
& u_{5}=f(x-\varepsilon, y-\varepsilon, z-\varepsilon, t), \\
& u_{6}=f(x-\varepsilon, y-\varepsilon, z-\varepsilon, t-\varepsilon), \\
& u_{7}=f(x-\lambda \varepsilon, y-\lambda \varepsilon, z-\lambda \varepsilon, t-\lambda \varepsilon),
\end{aligned}
$$

where $\varepsilon$ is any real constant.

\section{Symmetry Reductions and Exact Solutions of the Double Dispersion Equation}

In the previous sections, we obtained the infinitesimal generators $V_{i}(i=1,2, \cdots, 7)$. 
In this section, we will get similarity variables and its reduction equations, and we obtain similarity solutions and travelling wave solutions of Equation (10) by solving the reduction equations.

Case 1. For the infinitesimal generator $V_{1}=\frac{\partial}{\partial x}$, the similarity variables are $r=t, F(r)=\frac{1}{3} x-t u$, and the group-invariant solution is $u=\frac{\frac{1}{3} x-F(r)}{t}$, substituting the group-invariant solution into Equation (10), we obtain the following reduction equation

$$
F_{r}=0 \text {, }
$$

Obviously, $F=c_{1}$ is a solution of Equation (14), where $c_{1}$ is an arbitrary constant. Therefore, Equation (10) has a similarity solution as follows:

$$
u=\frac{\frac{1}{3} x-c_{1}}{t}
$$

where $p=3$.

Case 2. For the infinitesimal generator $V_{2}=\frac{\partial}{\partial y}$, the similarity variables are $r=t, F(r)=\frac{1}{3} y-t u$, and the group-invariant solution is $u=\frac{\frac{1}{3} y-F(r)}{t}$, substituting the group-invariant solution into Equation (10), we obtain the following reduction equation

$$
F_{r}=0 \text {, }
$$

Obviously, $F=c_{2}$ is a solution of Equation (16), where $c_{2}$ is an arbitrary constant. Therefore, Equation (10) has a similarity solution as follows:

$$
u=\frac{\frac{1}{3} y-c_{2}}{t}
$$

where $p=3$.

Case 3. For the infinitesimal generator $V_{3}=\frac{\partial}{\partial z}$, the similarity variables are $r=t, F(r)=\frac{1}{3} z-t u$, and the group-invariant solution is $u=\frac{\frac{1}{3} z-F(r)}{t}$, substituting the group-invariant solution into Equation (10), we obtain the following reduction equation

$$
F_{r}=0 \text {, }
$$

Obviously, $F=c_{3}$ is a solution of Equation (18), where $c_{3}$ is an arbitrary constant. Therefore, Equation (10) has a similarity solution as follows:

$$
u=\frac{\frac{1}{3} z-c_{1}}{t}
$$

where $p=3$. 
Case 4. For the infinitesimal generator $V_{4}=\frac{\partial}{\partial t}$, the similarity variables are $r=x+y+z, F(r)=u$, and the group-invariant solution is $u=F(r)$, substituting the group-invariant solution into Equation (10), we obtain the following reduction equation

$$
\Delta F(r)-\Delta^{2} F(r)+\Delta\left(F(r)^{p}\right)=0,
$$

Case 5. For the infinitesimal generator $V_{5}=\frac{\partial}{\partial x}+\frac{\partial}{\partial y}+\frac{\partial}{\partial z}$, the similarity variables are $F(r)=\frac{1}{3}(x+y+z)-t u$, and the group-invariant solution is $u=\frac{\frac{1}{3}(x+y+z)-F(r)}{t}$, substituting the group-invariant solution into Equation (10), we obtain the following reduction equation

$$
F_{r}=0 \text {, }
$$

Obviously, $F=c_{5}$ is a solution of Equation (21), where $c_{5}$ is an arbitrary constant. Therefore, Equation (10) has a similarity solution as follows:

$$
u=\frac{\frac{1}{3}(x+y+z)-c_{5}}{t}
$$

where $p=3$.

Case 6. For the infinitesimal generator $V_{6}=\frac{\partial}{\partial x}+\frac{\partial}{\partial y}+\frac{\partial}{\partial z}+\frac{\partial}{\partial t}$, the similarity variables are $r=x+y+z-\lambda t, F(r)=u$, and the group-invariant solution is $u=F(r)$, If we assume $k=0$ of Equation (10), substituting the group-invariant solution into Equation (10), we obtain the following reduction equation

$$
\left(1-\lambda^{2}\right) F_{r r r}-p(p-1) F^{p-2} F_{r}^{2}-p F^{p-1} F_{r r}+\left(\lambda^{2}-1\right) F_{r r}=0,
$$

So that Equation (23) is solvable in terms of Jacobi elliptic function, following the method described in [37].

If $\lambda^{2}=\frac{4}{3} \sqrt{\frac{c_{6}}{5}}+1, F(r)=\sqrt{5 c_{6}} d n^{2}\left(r, \sqrt{\frac{8}{5}}\right)$, where $d n\left(r, \sqrt{\frac{8}{5}}\right)$ is the Jacobi elliptic of the third kind function. Therefore, Equation (10) has a travelling wave solution as follows:

$$
u(x, y, z, t)=\sqrt{5 c_{6}} d n^{2}\left(x+y+z-\sqrt{\frac{4}{3} \sqrt{\frac{c_{6}}{5}}+1 t}, \sqrt{\frac{8}{5}}\right)
$$

where $p=2, c_{6}$ is an arbitrary constant.

If $\lambda^{2}=\frac{4}{3} \sqrt{\frac{c_{6}}{5}}+1, F(r)=\sqrt{5 c_{6}} c n^{2}\left(r, \sqrt{\frac{8}{5}}\right)$, where $c n\left(r, \sqrt{\frac{8}{5}}\right)$ is the Jacobi elliptic cosine function. Therefore, Equation (10) has a travelling wave solution as follows: 


$$
u(x, y, z, t)=\sqrt{5 c_{6}} c n^{2}\left(x+y+z-\sqrt{\frac{4}{3} \sqrt{\frac{c_{6}}{5}}}+1 t, \sqrt{\frac{8}{5}}\right)
$$

where $p=2, c_{6}$ is an arbitrary constant.

Case 7. For the infinitesimal generator $V_{7}=\left(\lambda \frac{\partial}{\partial x}+\frac{\partial}{\partial x}+\frac{\partial}{\partial x}\right)+\beta \frac{\partial}{\partial t}$, following the method described in [38], the similarity variables are $r=\beta(x+y+z)-\lambda t$, $F(r)=u$, and the group-invariant solution is $u=F(r)$, Substituting the group-invariant solution into Equation (10), we obtain the following reduction equation

$$
\begin{aligned}
& \left(\lambda^{2}-\beta^{2}\right) \beta^{2} F_{r r r}+k \lambda \beta^{2} F_{r r r}-\left(\lambda^{2}-\beta^{2}\right) \beta^{2} F_{r r} \\
& -\beta^{2} p F^{p-1} F_{r r}-\beta^{2} p(p-1) F^{p-2} F_{r}^{2}=0,
\end{aligned}
$$

Integrating twice with respect to $r$, we get

$$
\left(\lambda^{2}-\beta^{2}\right) \beta^{2} F_{r r}+k \lambda \beta^{2} F_{r}-\left(\lambda^{2}-\beta^{2}\right) F+\beta^{2} F^{p}=0,
$$

Let us assume that Equation (27) has solution of the form

$$
F=c_{7} H^{c_{8}}(r) \text {, }
$$

where $c_{7}, c_{8}$ are arbitrary constants, and $H(r)$ is a solution of the Jacobi equation

$$
H_{r}^{2}=\mu+r H^{2}+H^{4}
$$

If $H(r)=s n(r, m)$, where $s n(r, m)$ is Jacobi elliptic sine function, we obtain $F(r)=r s n(r, m)$ is a solution of Equation (27). By substituting $F(r)$ $=r s n(r, m)$ into (27), we obtain the equation

$$
\begin{aligned}
& -\beta^{2} r d n^{2}(r, m) \lambda^{2}-m \beta^{2} r c n^{2}(r, m) s n^{2}(r, m) \lambda^{2} \\
& -r s n(r, m) \lambda^{2}+\beta^{2} r c n^{2}(r, m) d n^{2}(r, m) s n^{-1}(r, m) \lambda^{2} \\
& -\beta^{2} r c n^{2}(r, m) d n^{2}(r, m) s n^{-1}(r, m) \lambda^{2}+k \beta^{2} r c n(r, m) d n(r, m) \\
& +\beta^{2} r s n(r, m)+m \beta^{4} r c n^{2}(r, m) n(r, m) \\
& -\beta^{4} r c n^{2}(r, m) d n^{2}(r, m) s n^{-1}(r, m) \\
& +\beta^{4} r c n^{2}(r, m) d n^{2}(r, m) s n^{-1}(r, m) \\
& +\beta^{2} r s n^{2}(r, m)=0,
\end{aligned}
$$

If $\lambda^{2}=2 \beta^{2}$, Equation (10) has a travelling wave solution as follows:

$$
u(x, y, z, t)=[\beta(x+y+z)-\lambda t] \operatorname{sn}(\beta(x+y+z)-\lambda t,-1),
$$

where $m=-1, p=2$. As $\operatorname{sn}(\beta(x+y+z)-\lambda t, 1)=\tanh (\beta(x+y+z)-\lambda t)$, we obtain $F(r)=\frac{1}{4} \tanh (r)$ is a solution of Equation (27), yb substituting $F(r)$ $=\frac{1}{4} \tanh (r)$ into Equation (27), we obtain a travelling wave solution as follows:

$$
u(x, y, z, t)=\frac{1}{4} \tanh \left(x+y+z-\frac{t}{2}\right)
$$


where $k=8, p=3$

If $F(r)=r c n(r, m)$ is a solution of Equation (27). By substituting $F(r)$ $=r c n(r, m)$ into (27), when $\lambda^{2}=2 \beta^{2}$, Equation (10) has a travelling wave solution as follows:

$$
u(x, y, z, t)=[\beta(x+y+z)-\lambda t] c n(\beta(x+y+z)-\lambda t,-1),
$$

where $m=1, p=2$

\section{Conclusion}

In this paper, we study the symmetry reductions and explicit solutions of a multidimensional double dispersion equation by means of classical Lie group method. First, we get the symmetry groups and the infinitesimal generators of Equation (10). Then, we discuss the Lie symmetry groups of the multidimensional double dispersion equation and obtain the group-invariant solution. Finally, we obtain similarity solutions and travelling wave solutions of Equation (10) using similarity variables.

\section{Acknowledgments}

This work was supported by the Natural Science Foundation of Education of Guizhou Province: KY[2016]103.

\section{References}

[1] Samsonov, A.M. (2001) Strain Solitons in Solids and How to Construct Them. Chapman and Hall, Boca Raton. https://doi.org/10.1201/9781420026139

[2] Porubov, A.V. (2003) Amplification of Nonlinear Strain Waves in Solids. World Scientific, Singapore. https://doi.org/10.1142/5238

[3] Christov, C.I., Maugin, G.A. and Porubov, A.V. (2007) On Boussinesq's Paradigm in Nonlinear Wave Propagation. Comptes Rendus Mecanique, 335, 521-535. https://doi.org/10.1016/j.crme.2007.08.006

[4] Engelbrecht, J., Salupere, A. and Tamm, K. (2011) Waves in Microstructured Solids and the Boussinesq Paradigm. Wave Motion, 48, 717-726.

[5] Erbay, H.A., Erbay, S. and Erkip, A. (2015) Existence and Stability of Traveling Waves for a Class of Nonlocal Nonlinear Equations. Journal of Mathematical Analysis and Applications, 425, 307-336.

[6] Erbay, H.A., Erbay, S. and Erkip, A. (2016) Instability and Stability Properties of Traveling Waves for the Double Dispersion Equation. Nonlinear Analysis, 133, 1-14.

[7] Pasternak, N.L. (1954) New Method for Calculation of Foundation on the Elastic Basement. Gosstroiizdat, Moscow. (In Russian)

[8] Samsonov, A.M. and Sokurinskaya, E.V. (1989) Energy Exchange between Nonlinear Waves in Elastic Waveguides and External Media. In: Engelbrecht, P.J., Ed., Nonlinear Waves in Active Media, Springer, Berlin, 99-104. https://doi.org/10.1007/978-3-642-74789-2_13

[9] Samsonov, A.M. (1994) Nonlinear Strain Waves in Elastic Waveguide. In: Jeffrey, A. and Engelbrecht, J., Eds., Nonlinear Waves in Solids, CISM Courses and Lectures, Vol. 341, Springer, Wien, 349-382. https://doi.org/10.1007/978-3-7091-2444-4_6

[10] Samsonov, A.M. (1988) On Existence of Longitudinal Strain Solitons in an Nonli- 
nearly Elastic Rod. Soviet Physics-Doklady, 4, 298-300.

[11] Samsonov, A.M. (1993) On Some Exact Travelling Wave Solutions for Nonlinear Hyperbolic Equation. In: Fusco, D. and Jeffrey, A., Eds., Nonlinear Waves and Dissipative Effects, Pitmann Research Notes in Mathematics Series, Vol. 227, Longman Scientific Technical, Longman, 123-132.

[12] Chen, G., Wan, Y. and Wang, S. (2004) Initial Boundary Value Problem of the Generalized Cubic Double Dispersion Equation. Journal of Mathematical Analysis and Applications, 299, 563-577.

[13] Wang, S. and Chen, G. (2006) Cauchy Problem of the Generalized Double Dispersion Equation. Nonlinear Analysis, 64, 159-173.

[14] Polat, N. and Ertas, A. (2009) Existence and Blow-Up of Solution of Cauchy Problem for the Generalized Damped Multidimensional Boussinesq Equation. Journal of Mathematical Analysis and Applications, 349, 10-20.

[15] Yang, Z., Feng, N. and Ma, T.T. (2015) Global Attractor for the Generalized Double Dispersion Equation. Nonlinear Analysis, 115, 103-116.

[16] Babaoglu, C., Erbay, H.A. and Erkip, A. (2013) Global Existence and Blow-Up of Solutions for a General Class of Doubly Dispersive Nonlocal Nonlinear Wave Equations. Nonlinear Analysis, 77, 82-93.

[17] Chen, G.W. and Xue, H.X. (2008) Periodic Boundary Value Problem and Cauchy Problem of the Generalized Cubic Double Dispersion Equation. Acta Mathematica Scientia, 28, 573-587.

[18] Kato, M., Wang, Y.Z. and Kawashima, S. (2013) Asymptotic Behavior of Solutions to the Generalized Cubic Double Dispersion Equation in One Space Dimension. Kinetic and Related Models, 6, 969-987. https://doi.org/10.3934/krm.2013.6.969

[19] Kawashima, S. and Wang, Y.Z. (2015) Global Existence and Asymptotic Behavior of Solutions to the Generalized Cubic Double Dispersion Equation. Analysis and Applications, 13, 233-254.

[20] Liu, Y.C. and Xu, R.Z. (2008) Potential Well Method for Cauchy Problem of Generalized Double Dispersion Equations. Journal of Mathematical Analysis and Applications, 338, 1169-1187.

[21] Liu, Y.C. and Xu, R.Z. (2008) Potential Well Method for Initial Boundary Value Problem of the Generalized Double Dispersion Equations. Communications on Pure and Applied Mathematics, 7, 63-81.

[22] Wang, S.B. and Da, F. (2013) On the Asymptotic Behaviour of Solution for the Generalized Double Dispersion Equation. Applied Analysis, 92, 1179-1193. https://doi.org/10.1080/00036811.2012.661044

[23] Xu, R.Z., Liu, R.Z. and Liu, Y.C. (2009) Global Existence and Nonexistence of Solution for Cauchy Problem of Multidimensional Double Dispersion Equations. Journal of Mathematical Analysis and Applications, 359, 739-751.

[24] Xu, R.Z., Liu, Y.C. and Yu, T. (2009) Global Existence of Solution for Cauchy Problem of Multidimensional Generalized Double Dispersion Equations. Nonlinear Analysis. Theory, Methods \& Applications, 71, 4977-4983.

[25] Dai, Z.D. and Guo, B.L. (1999) Long Time Behavior of Nonlinear Strain Waves in Elastic Waveguides. Journal of Partial Differential Equations, 12, 301-312.

[26] Dai, Z.D. and Guo, B.L. (2000) Global Attractor of Nonlinear Strain Waves in Elastic Waveguides. Acta Mathematica Scientia, 20, 322-334.

[27] Yang, Z.J. and Li, K. (2013) Longtime Dynamics for a Elastic Waveguide Model. Discrete and Continuous Dynamical Systems Series S, 797-806. 
[28] Tian, C. (2001) Applications of Lie Groups to Differential Equations. Science Press, Beijing.

[29] Wang, M.L., Zhou, Y. and Li, Z. (1996) Application of a Homogeneous Balance Method to Exact Solutions of Nonlinear Equations in Mathematical Physics. Physics Letters $A, 216,67-75$.

[30] Fan, E. (2000) Extended Tanh-Function Method and Its Applications to Nonlinear Equations. Physics Letters A, 277, 212-218.

[31] Bluman, J.C.W. and Cole, J.D. (1974) Similarity Methods for Differential Equations. Springer, Berlin. https://doi.org/10.1007/978-1-4612-6394-4

[32] Olver, P.J. (2000) Applications of Lie Groups to Differential Equations. Springer Verlag, Berlin.

[33] Liu, H., Li, J. and Liu, L. (2009) Lie Group Classifications and Exact Solutions for Two Variable-Coefficient Equations. Applied Mathematics and Computation, 215, 2927-2935.

[34] Stephani, H. and MacCallum, M. (1989) Differential Equations: Their Solution Using Symmetries. Cambridge University Press, Cambridge.

[35] Cantwell, B. (2002) Introduction to Symmetry Analysis. Cambridge University Press, Cambridge.

[36] Clarkson, P.A. and Priestley, T.J. (1996) Symmetries of a Generalised Boussinesq Equation. Electronic Edition.

[37] Bruzón, M.S. and Gandarias, M.L. (2008) Similarity Reductions of a Generalized Double Dispersion Equation. 79th Annual Meeting of the International Association of Applied Mathematics and Mechanics, 8, 10587-10588,. https://doi.org/10.1002/pamm.200810587

[38] Bruzón, M.S. and Gandarias, M.L. (2009) Travelling Wave Solutions for a Generalized Double Dispersion Equation. Nonlinear Analysis, 71, 2109-2117.

Submit or recommend next manuscript to SCIRP and we will provide best service for you:

Accepting pre-submission inquiries through Email, Facebook, LinkedIn, Twitter, etc. A wide selection of journals (inclusive of 9 subjects, more than 200 journals)

Providing 24-hour high-quality service

User-friendly online submission system

Fair and swift peer-review system

Efficient typesetting and proofreading procedure

Display of the result of downloads and visits, as well as the number of cited articles

Maximum dissemination of your research work

Submit your manuscript at: http://papersubmission.scirp.org/

Or contact am@scirp.org 